\section{The association of maternal factors with epibulbar dermoid of newborn: a retrospective, matched case-control study}

$\mathrm{S} \mathrm{Wu}^{1,4}, \mathrm{Y} \mathrm{Fan}^{2,4}, \mathrm{D} \mathrm{Wu^{1 }}, \mathrm{J} \mathrm{Hong}^{1,3}$ and $\mathrm{J} X \mathrm{u}^{1}$

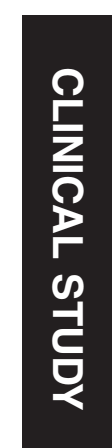

affect microvascular development and predispose the fetus to future ED. Eye (2017) 31, 1099-1105; doi:10.1038/eye.2017.40; published online 24 March 2017

\section{Introduction}

Epibulbar dermoids (EDs) are congenital, benign tumors involving the cornea and subconjuntiva, occasionally invading the anterior chamber. ${ }^{1}$ As a subtype of epibulbar choristomas, the tumor consists of a combination of ectodermal and mesodermal elements including hair, fat, keratinized epithelium, sebaceous glands, fibrous tissue, and blood vessels. Epibulbar choristomas can be observed in one-three of 10000 newborns, ${ }^{2}$ and EDs are the most common epibulbar choristomas ${ }^{3}$ and, moreover, the most common ocular congenital tumors. ${ }^{4}$ EDs may hinder the visual development of newborns and cause visual impairment and cosmetic problems if untreated. ${ }^{5}$ For example, a recent study containing eight children with median-sized ED showed that all the participants had astigmatism. ${ }^{6}$ Surgical removal of the tumors along with corneal transplantation, therefore, is indicated. ${ }^{3}$

The etiology of ED is obscure. Paired Box 6 (PAX6) is a transcription factor that plays a key role in limbal stem cells and corneal epithelial fate determination and maintenance. 7,8 A recent study showed that PAX6 was completely absent from corneal dermoid tissue, suggesting its association with the pathogenesis of corneal dermoids, a subtype of $\mathrm{ED} ;{ }^{9}$ nicotine is a potential environmental factor associated with this PAX6 absence. ${ }^{10}$ Concerning genetic factors, most ED cases are considered sporadic. However, several hereditary forms of ED have been reported, including ring
${ }^{1}$ Department of Ophthalmology and Visual Science, Eye, Ear, Nose, and Throat Hospital, Shanghai Medical College, Fudan University, Shanghai, China

${ }^{2}$ Department of Nursing, Eye, Ear, Nose, and Throat Hospital, Shanghai Medical College, Fudan University, Shanghai, China

${ }^{3}$ Schepens Eye Research Institute, Massachusetts Eye and Ear Infirmary, Harvard Medical School, Boston, MA, USA

Correspondence:

J Xu, Department of Ophthalmology and Visual Science, Eye, Ear, Nose, and Throat Hospital, Shanghai Medical College, Fudan University, 83 Fenyang Road, Shanghai 200031, China Tel/Fax: 8613816881607. E-mail: jianjiangxu@ 126.com

${ }^{4}$ These authors contributed equally to this work and are considered first authors.

Received: 12 July 2016 Accepted in revised form: 31 January 2017 Published online: 24 March 2017 
dermoids, ${ }^{11}$ chromosome Xq24-qter corneal dermoids, ${ }^{12}$ Goldenhar syndrome, ${ }^{13}$ and trisomy 8 mosaic syndrome. ${ }^{14,15}$

In contrast with the progress in understanding genetic risk factors in recent years, little is known about the environmental risk factors for ED. Oculo-auriculovertebral spectrum is a complex and heterogeneous condition that encompasses a variety of defects in structures derived from the first and second branchial arches, including Goldenhar syndrome with ED. ${ }^{13}$ A population-based case-control study conducted in Spain indicated that oculo-auriculo-vertebral spectrum had a higher incidence in the infants of diabetic mothers than in the general population. ${ }^{16}$ However, these results are only weakly associated with risk of ED, because Goldenhar syndrome is relatively rare and only comprises a small proportion of ED cases.

The current study attempted to investigate the potential association of maternal and paternal factors and exposure during pregnancy with ED in a large and well-defined group of patients who were diagnosed and followed at a national medical center. Nicotine exposure and maternal diabetes mellitus were included, as well as other factors related to congenital malformations derived from the same embryotic origin (ectoderm and mesoderm) as ED. ${ }^{17-23}$

\section{Methods}

This study had a paired case-control design. ${ }^{24}$ A total of 121 Chinese children with ED who came consecutively to the Department of Ophthalmology and Vision Science at the Eye and ENT Hospital, School of Shanghai Medicine, Fudan University, Shanghai, China (Eye \& ENT Hospital) from 2014 to 2015 were initially considered for this study. ED was diagnosed by two clinicians from the hospital. All of the patients' parents agreed to participate, and attended an appointment to be interviewed. A total of 140 Chinese children without ED were invited as the control group following identification through community health records. All the control children and their parents lived in a large community in Shanghai. Because of financial reasons, we did not invite the patients' relatives from various other places in China to come to Shanghai to be interviewed. The parents of two $(1.4 \%)$ of the children declined to participate, and the parents of three $(2.1 \%)$ children did not attend the appointment. All the remaining children were enrolled, born during the past 7 years. Because ED is a congenital malformation that occurs at birth, we did not age match the case and control children.

The questionnaire was administered by a researcher who was not part of the team of clinicians diagnosing and treating the patients of ED in a blinded fashion. The mothers of patients were interviewed after the diagnostic work-up was finished, but they were not informed of the possible association between any factor and ED during routine visits. To limit the recall bias, we also reviewed prenatal logbooks, and records of serological, blood pressure, hemoglobin, and blood sugar tests. The medical records were copies provided by the mothers.

The structured questionnaire contained four main sections. The first section concerned the basic information and personal history of the mother (pregnancy age, diabetes mellitus history, gestation history, miscarriage history, and hepatic virus B carrying history). The second section focused on exposure to maternal diseases, and symptoms and corresponding medical treatments during pregnancy that could potentially be risk factors for congenital anomalies of the newborn; these included pregnancy-induced hypertension, gestational diabetes mellitus, anemia, common cold, low serum progesterone in the first trimester, threatened miscarriage, antibiotic medication, and progesterone medication. The definitions of these health statuses were stated in the Supplementary Appendix. The time of disease occurrence and duration was also recorded. The third section focused on maternal environmental exposures, including tobacco smoking, paternal smoking, and exposure to formaldehyde, pesticides, and X-rays during pregnancy. All the mothers were asked to describe their work place and their house, especially from the following aspect: whether the place was newly decorated or whether it was close to farming land; how long and how often they stayed in there; whether there were other ways that could potentially lead the mothers to formaldehyde or pesticide exposure. All the parents were asked about whether he or she smoked before and during maternal pregnancy, what the smoking frequency and smoke loading were, whether there was a period ( $>1$ month) in which he or she did not smoke at all, and whether the couples live together. In addition, records of X-ray test, for any part of the body, were asked about and reviewed from the logbook. The last section was to collect information on parental socioeconomic status, based on the real-time Prasad's social classification scale $^{25}$ including median education level, occupation status, and monthly family income. To balance the regional differences of consumption levels, the monthly family income classification was adjusted with domestic consumer price indexes obtained through the online database on the official website of National Bureau of Statistics of China.

The case and control participants were designed to be case matched for sex, gestational age ( $<1$ week apart), birth weight ( $<100 \mathrm{~g}$ difference), and parental socioeconomic status level. Fourteen control participants were excluded for the statistical analysis because of match failure. Thus, 121 case-control pairs remained. 
The study was conducted in the Department of Ophthalmology and Vision Science at the Eye and ENT Hospital in accordance with the tenets of the Declaration of Helsinki, and was approved by the ethics committee of the Eye and ENT Hospital. As all the participants were under 18 years old, the mothers of the participants signed an informed consent form. Privacy was preserved through using alphanumerical code identifiers.

Data were analyzed using IBM SPSS Statistics, version 19.0 (IBM, Armonk, NY, USA) and PASS version 11.0.7 (NCSS, LLC, Kaysville, UT, USA). A $P$-value of $<0.05$ was considered significant. The unpaired $t$-test was used to analyze the difference in pregnancy age of the mothers. For categorical variables, crude odd ratios (ORs) were calculated using conditional asymptotic or exact univariate logistic regression models, depending on the variable itself. A multivariate conditional logistic regression model was adapted to include the matching effect. The variable selection method for the model was back elimination, and was based on the algorithm by Hosmer and Lemeshow. ${ }^{26}$ The values of $\exp (\mathrm{B})$, indicated as odd ratios, were shown with the corresponding 95\% confidence intervals and statistical significance.

Collinearity was analyzed via $\chi^{2} 2 \times 2$ test within the total group. In addition, power tests were performed to identify whether the sample size was large enough. The value of $\varphi$ was set to be 0.15 . A power value of 0.8 or more was recognized as a high calculation power.

\section{Results}

Table 1 summarizes the ED patient characteristics. Of the 121 patients, two had Goldenhar syndrome, and two mothers of the recruited patients were found to have undergone X-ray test for chest during pregnancy. No statistically significant difference was found in the median pregnancy age between mothers of case and control participants $(27.73 \pm 3.90$ (SD) vs $29.67 \pm 3.45, P=0.152)$ and also in the median age between the two groups of children (2.44 \pm 1.99 , ranging $0.5-6$ vs $2.95 \pm 1.75$, ranging $0.5-7, P=0.898)$. Mothers who caught common cold during pregnancy were divided into three following subgroups: those who caught common cold during first, second, and the last trimester of pregnancy. Low progesterone was found only during the first trimester among the recruited mothers. In addition, none of the mothers smoked, and all the fathers that were identified as smokers smoked before maternal pregnancy and throughout the whole pregnancy period. There was a significantly higher prevalence of history of maternal inevitable miscarriage $(P=0.008)$, exposure to the common cold in the first trimester $(P<0.001)$, and paternal smoking $>$ half a pack (half a pack $=10$ cigarettes) per day $(P=0.004)$ in case children compared
Table 1 Characteristics of 121 patients with ED

\begin{tabular}{|c|c|}
\hline Characteristic & Patients $^{\mathrm{a}}$ \\
\hline Female sex & $51(42)$ \\
\hline Birth weight in $\mathrm{kg}$, mean $\pm \mathrm{SD}$ & $3.29 \pm 0.50$ \\
\hline Gestational age in weeks, mean $\pm S D$ & $38.77 \pm 1.37$ \\
\hline In vitro fertilization neonate & $1(1)$ \\
\hline Diameter of $\mathrm{ED}$, mean $\pm \mathrm{SD}, \mathrm{mm}$ & $3.63 \pm 1.44$ \\
\hline \multicolumn{2}{|l|}{ ED localization } \\
\hline Unilateral & $117(97)$ \\
\hline Right & $55(45)$ \\
\hline Left & $62(52)$ \\
\hline Bilateral & $4(3)$ \\
\hline Inferior temporal & $97(81)$ \\
\hline Superior temporal & $1(1)$ \\
\hline Inferior nasal & $1(1)$ \\
\hline Superior nasal & $1(1)$ \\
\hline Temporal & $17(14)$ \\
\hline Nasal & $1(1)$ \\
\hline Inferior & $2(2)$ \\
\hline Cornea involvement & $120(99)$ \\
\hline Pupil interrupted ${ }^{\mathrm{b}}$ & $6(5)$ \\
\hline Limbus involvement & $102(84)$ \\
\hline Bulbar conjunctiva involvement & $97(80)$ \\
\hline Isolated ED & $65(54)$ \\
\hline \multicolumn{2}{|l|}{ Additional associated findings } \\
\hline Accessory auricle & $53(43)$ \\
\hline Unilateral & $39(32)$ \\
\hline Bilateral & $14(12)$ \\
\hline Atresia auris & $1(1)$ \\
\hline Hemifacial microsomia & $1(1)$ \\
\hline Vertebral scoliosis & $2(2)$ \\
\hline Cleft lip & $1(1)$ \\
\hline Atrial septal defect ${ }^{c}$ & $1(1)$ \\
\hline Hexadactylia & $1(1)$ \\
\hline Contracture of thumb tendon sheath & $1(1)$ \\
\hline \multicolumn{2}{|l|}{ Family history $^{\mathrm{d}}$} \\
\hline ED & $2(2)$ \\
\hline Accessory auricle & $8(7)$ \\
\hline \multicolumn{2}{|l|}{ SES level } \\
\hline I & $16(13)$ \\
\hline II. & $39(32)$ \\
\hline III & $44(36)$ \\
\hline IV & $17(14)$ \\
\hline $\mathrm{V}$ & $5(4)$ \\
\hline
\end{tabular}

Abbreviations: ED, epibulbar dermoid; SD, standard deviation; SES, socioeconomic status. ${ }^{a}$ Values are numbers (percentages) unless otherwise indicated. ${ }^{b}$ No mydriatic was used. 'Diagnosed with echocardiography. ${ }^{\mathrm{d} O n l y}$ lineal relatives were included.

with control children. In addition, mothers of the case children had a significantly greater prevalence of progesterone medication in the first trimester $(P=0.020)$. There was no significant difference between the mothers of case and control children in the prevalence of other variables including pregnancy-induced hypertension, gestational diabetes mellitus, anemia, low serum progesterone, threatened miscarriage, antibiotic 
Table 2 Association of prevalence of maternal risk factors with ED in newborns

\begin{tabular}{|c|c|c|c|c|c|}
\hline Maternal risk factor & $\begin{array}{l}\text { Mothers of case } \\
\text { participants, } \mathrm{n}(\%)\end{array}$ & $\begin{array}{l}\text { Mothers of control } \\
\text { participants, } \mathrm{n}(\%)\end{array}$ & $\begin{array}{l}\text { Crude OR } \\
(95 \% \text { CI) }\end{array}$ & P-value & Power \\
\hline History of $\mathrm{DM}^{\mathrm{a}}$ & $2(2)$ & $0(0)$ & - & 0.499 & - \\
\hline History of inevitable miscarriage & $35(29)$ & $19(15)$ & $2.33(1.23-4.40)$ & 0.008 & 0.638 \\
\hline History of carrying $\mathrm{HBV}^{\mathrm{a}}$ & $3(2)$ & $2(2)$ & $1.51(0.25-9.22)$ & 1.000 & 0.069 \\
\hline $\mathrm{PIH}^{\mathrm{a}}$ & $4(3)$ & $2(2)$ & $2.03(0.37-11.32)$ & 0.684 & 0.112 \\
\hline $\mathrm{GDM}^{\mathrm{a}}$ & $2(2)$ & $6(5)$ & $0.32(0.06-1.63)$ & 0.281 & 0.246 \\
\hline Anemia during pregnancy & $12(10)$ & $11(9)$ & $1.10(0.47-2.60)$ & 0.827 & 0.054 \\
\hline Common cold during pregnancy & $53(44)$ & $32(21)$ & $2.17(1.26-3.72)$ & 0.005 & 0.681 \\
\hline Common cold in the first trimester & $39(32)$ & $13(11)$ & $3.95(1.98-7.88)$ & $<0.001$ & 0.973 \\
\hline Common cold in the second trimester & $12(10)$ & $14(12)$ & $0.84(0.37-1.90)$ & 0.678 & 0.067 \\
\hline Common cold in the third trimester ${ }^{\mathrm{a}}$ & $2(2)$ & $4(2)$ & $0.49(0.09-2.74)$ & 0.408 & 0.078 \\
\hline $\begin{array}{l}\text { Low serum progesterone in the first trimester } \\
\text { (without threatened miscarriage) } \text { ( }^{\mathrm{a}}\end{array}$ & $10(8)$ & $5(4)$ & $2.09(0.69-6.31)$ & 0.286 & 0.200 \\
\hline Threatened miscarriage $^{a}$ & $9(7)$ & $2(2)$ & $4.78(1.01-22.61)$ & 0.059 & 0.515 \\
\hline Antibiotic medication during pregnancy ${ }^{\mathrm{a}}$ & $6(5)$ & $3(2)$ & $2.05(0.50-8.40)$ & 0.499 & 0.114 \\
\hline Progesterone medication in the first trimester & $18(15)$ & $7(6)$ & $2.85(1.14-7.09)$ & 0.020 & 0.510 \\
\hline Maternal smoking & $0(0)$ & $0(0)$ & - & - & - \\
\hline Paternal smoking & $61(50)$ & $40(33)$ & $2.06(1.22-3.46)$ & 0.006 & 0.708 \\
\hline Paternal smoking ( $\leq$ half a pack per day) & $35(29)$ & $27(22)$ & $1.42(0.79-2.53)$ & 0.239 & 0.186 \\
\hline Paternal smoking ( $>$ half a pack per day) & $26(21)$ & $10(8)$ & $3.04(1.39-6.62)$ & 0.004 & 0.731 \\
\hline Formaldehyde exposure during pregnancy ${ }^{a}$ & $7(6)$ & $3(2)$ & $2.42(0.61-9.57)$ & 0.333 & 0.237 \\
\hline Pesticide exposure during pregnancy ${ }^{\mathrm{a}}$ & $3(2)$ & $0(0)$ & - & 0.247 & - \\
\hline X-ray exposure during pregnancy ${ }^{\mathrm{a}}$ & $2(2)$ & $0(0)$ & - & 0.499 & - \\
\hline
\end{tabular}

Abbreviations: CI, confidence intervals; DM, diabetes mellitus; GDM, gestational diabetes mellitus; OR, odds ratio; PIH, pregnancy-induced hypertension.

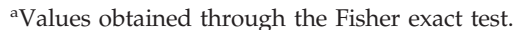

Table 3 Association between maternal factors and ED of the newborn in a multivariate conditional logistic model

\begin{tabular}{|c|c|c|}
\hline Variable & OR $(95 \% C I)$ & P-value \\
\hline History of inevitable miscarriage & $2.59(1.13-5.90)$ & 0.023 \\
\hline Common cold in the first trimester & $3.94(1.74-8.93)$ & 0.001 \\
\hline Low serum progesterone in the first trimester (without threatened miscarriage) & $0.39(0.03-5.55)$ & 0.488 \\
\hline Threatened miscarriage & $1.06(0.05-22.81)$ & 0.972 \\
\hline Progesterone medication in the first trimester & $1.82(0.15-22.51)$ & 0.641 \\
\hline \multicolumn{3}{|l|}{ Paternal smoking } \\
\hline Paternal smoking ( $\leq$ half a pack per day) & $1.59(0.81-3.12)$ & 0.174 \\
\hline Paternal smoking ( $>$ half a pack per day) & $4.81(1.74-13.28)$ & 0.002 \\
\hline
\end{tabular}

Abbreviations: $\mathrm{CI}$, confidence intervals; $\mathrm{OR}$, odds ratio.

medication, and exposure to formaldehyde, pesticides, and X-rays during pregnancy (Table 2). The statistical power of the tests for history of inevitable miscarriage, common cold in the first trimester were high $(>0.8)$, while the powers of the tests for the other factors listed were not.

In the multivariate conditional logistic regression model, the factors significantly associated with ED were: history of maternal inevitable miscarriage $(P=0.023)$, exposure to the common cold in the first trimester $(P=0.001)$, and paternal smoking $>$ half a pack per day during pregnancy $(P=0.002$, Table 3$)$. The other covariates used in the model, including low serum progesterone, paternal smoking $\leq$ half a pack per day, threatened miscarriage, and progesterone medication, were not significantly associated with ED. The factor with the greatest statistical significance and the greatest odds ratio was paternal smoking $>$ half a pack per day.

No significant collinearity was found between either two of the three identified potential risk factors from the total group (Table 4). However, all the calculation power values were lower than 0.1 .

\section{Discussion}

Our results suggest that maternal history of inevitable miscarriage, common cold in the first trimester, and exposure to paternal smoking ( $>$ half a pack per day) during pregnancy were associated with an increased risk of ED for the newborn; in contrast, maternal exposure to 
Table 4 Collinearity analysis between the identified potential risk factors

\begin{tabular}{|c|c|c|c|}
\hline Items & $\chi^{2}$ & P-value & Power \\
\hline Paternal smoking $>$ half a pack per day and maternal miscarriage history & 0.003 & 0.960 & 0.050 \\
\hline Paternal smoking $>$ half a pack per day and maternal common cold in the first trimester & 0.105 & 0.746 & 0.062 \\
\hline Maternal miscarriage history and common cold in the first trimester & 0.372 & 0.542 & 0.093 \\
\hline
\end{tabular}

other factors including pesticides and X-rays known to possibly predispose to congenital anomalies, maternal diabetes mellitus history, and gestational diabetes mellitus were not significantly associated with ED. However, the above-mentioned median or low power of some of the tests means that the results indicating insignificant associations should be conservatively interpreted. Maternal exposure to the common cold during the second and third trimesters was not significantly associated with ED, supporting the previous finding that first trimester maternal influenza exposure was associated with an increased risk of many congenital anomalies. ${ }^{27}$ Paternal smoking of no $>$ half a pack per day was not significantly associated with ED, suggesting a dose-dependent effect of tobacco. In multivariate analysis, all three factors remained significant after considering all potential confounders assessed in this study. Of note, two of the three factors (miscarriage and smoking) showed greater ORs in the multivariate logistic regression model than those in the univariate models. Paternal smoking $>$ half a pack per day yielded the greatest odds ratio among the three significant environmental factors, reemphasizing the importance of this risk factor for congenital anomalies according to previous studies. ${ }^{28-30}$

Several studies have indicated that previous miscarriage could indicate a higher risk for poor outcomes in subsequent pregnancies, ${ }^{31-33}$ especially those due to placental causes. ${ }^{33}$ This might be related to a higher possibility of placental inflammation for pregnant woman with a history of pregnancy loss, ${ }^{34}$ which may also trigger a fetal inflammatory response and vasculopathy. ${ }^{35}$ Maternal common cold and smoke exposure during pregnancy are also possibly linked with fetal inflammation and abnormal vascular development. ${ }^{36-38}$ These factors might potentially affect the differentiation of surface ectoderm and mesoderm during embryonic eye development. Moreover, a recent study found that nicotine could induce CpG methylation of a PAX6-binding motif, and thus inhibit downstream steroid hormone production essential to antiinflammatory effect and fetal development; ${ }^{10}$ this might potentially correlate to the PAX6 loss identified by Li et al. ${ }^{39}$ However, the exact mechanisms still remain unknown and need to be further investigated. Paternal smoking might also genetically affect sperm; ${ }^{29}$ although little evidence is provided for this possible mechanism, it may offer a pathway to further investigate the genetic factors for ED in the future.

To our knowledge, this is the first study to investigate maternal risk factors for ED. We used a paired casecontrol design to maximize the likelihood that maternal factors were the only different variables for the participants and therefore should be the key determinants affecting whether neonates develop ED. Concerning the interaction among the significant factors, it is universally acknowledged that secondhand smoke exposure can increase the risk of respiratory diseases. ${ }^{40}$ Smoke exposure is also a risk factor for miscarriage. ${ }^{41}$ The sample size of our study was not sufficient to investigate whether there was an interaction or synergistic action among miscarriage history, the common cold, paternal smoking, and even genetic factors. This issue deserves further exploration.

This study had some limitations. First, because of the case-control and questionnaire-based approach, all assessments were based only on retrospective histories, and mothers of participants may have had difficulty ascertaining factors or exposures to specific agents. Although we reviewed the prenatal logbooks and laboratory test records as objective evidence, some maternal exposures, such as exposure to formaldehyde and pesticides, relied solely on the questionnaire answers, which could have potentially resulted in recall bias. Second, we did not investigate whether the fathers were using a nicotine replacement program during the pregnancy. Also, we are not able to identify whether the common cold was associated with influenza. Third, nutritional factors, which are associated with socioeconomic backgrounds, were also not included. Finally, the case participants came from various places in China, whereas the control group lived in Shanghai, so the two groups were not region matched. Therefore, the socioeconomic match in our study might be insufficient. It is possible that these factors could have influenced the results. This issue needs to be considered further in future research.

Although treating ED of newborns is primarily a mission for ophthalmological surgeons, the findings of this study are relevant to a much broader audience for ED as the most common congenital ocular tumor. Our study found that almost half of the ED newborns had other associated findings, especially accessory auricles, which is consistent with the previous findings that ED is not 
isolated in some occasions and many ED newborns could also be found to have accessory auricles. ${ }^{42}$ Therefore, physicians, especially pediatricians and obstetricians, in outpatient settings have a major role in identifying potentially at-risk fetuses and providing early, primary prevention of ED. They should also provide counseling and advice on smoking cessation to fathers. Other physicians, including otologists, also have a role in treating associated findings such as accessory auricles. For some factors, such as pregnancy-induced hypertension, gestational diabetes mellitus, and formaldehyde exposure, the sample size of our study is too small to achieve a significant and meaningful result. Therefore, more research is necessary to further elucidate the risk factors and pathogenesis of ED, and this study is the first step to larger and more carefully controlled case-control studies that will continue this investigation. Also, with the current study linking paternal smoking to development of $\mathrm{ED}$, and the knowledge we have of the effects of nicotine on molecular regulation, it would be worthwhile to evaluate ocular surface and anterior segment development in the eye of affected infants. We hope this study urges stronger counseling for miscarriage and for stopping smoking in husbands of pregnant women, and more aggressive programs to reduce secondhand smoke pollution.

\section{Summary}

What was known before

- Genetic factors that predispose infants to ED have been studied, while maternal factors that may be associated with future risk for ED are less clear.

\section{What this study adds}

- Through a case-control design, we determined that maternal miscarriage, common cold exposure in first trimester, and paternal smoking during pregnancy may be significant risk factors for ED in newborns. Our results highlight the importance of secondhand smoking prevention in pregnancy.

\section{Conflict of interest}

The authors declare no conflict of interest.

\section{Acknowledgements}

The authors thank Ms Xiaoyan Li for her contribution in recruiting participants for this study. This study is supported by grants from the Key Clinic Medicine Research Program, the Ministry of Health, China (201302015); the National Science and Technology Research Program, the Ministry of Science and Technology, China (2012BAI08B01); the National Natural
Science Foundation of China (81170817, 81200658, 81300735, 81270978, U1205025, and 81330022); the Scientific Research Program, Science and Technology Commission of Shanghai Municipality, Shanghai (13441900900, 13430720400); the Chinese Postdoctoral Fund (XMU135890); and the New Technology Joint Research Project in Shanghai Hospitals (SHDC12014114). The sponsors or funding organizations had no role in the design or conduct of this research.

\section{References}

1 Golubovic S, Latkovic Z, Horvatic-Obradovic M. Surgical treatment of large corneal dermoid. Doc Ophthalmol 1995; 91: 25-32.

2 Elsas FJ, Green WR. Epibulbar tumors in childhood. Am J Ophthalmol 1975; 79: 1001-1007.

3 Pirouzian A. Management of pediatric corneal limbal dermoids. Clin Ophthalmol 2013; 7: 607-614.

4 Nevares RL, Mulliken JB, Robb RM. Ocular dermoids. Plast Reconstr Surg 1988; 82: 959-964.

5 Robb RM. Astigmatic refractive errors associated with limbal dermoids. J Pediatr Ophthalmol Strabismus 1996; 33: 241-243.

6 Wu KI, Chu HS, Pai AS, Hou YC, Lin SY, Chen WL et al. Surgical management of limbal dermoids using anterior corneal buttons from descemet stripping automated endothelial keratoplasty donor tissue as patch grafts. Cornea 2017; 36: 64-67.

7 Ouyang H, Xue Y, Lin Y, Zhang X, Xi L, Patel S et al. WNT7A and PAX6 define corneal epithelium homeostasis and pathogenesis. Nature 2014; 511: 358-361.

8 Li W, Chen YT, Hayashida Y, Blanco G, Kheirkah A, $\mathrm{He} \mathrm{H}$ et al. Down-regulation of Pax6 is associated with abnormal differentiation of corneal epithelial cells in severe ocular surface diseases. J Pathol 2008; 214: $114-122$.

9 Li G, Xu F, Zhu J, Krawczyk M, Zhang Y, Yuan J et al. Transcription factor PAX6 (Paired Box 6) controls limbal stem cell lineage in development and disease. J Biol Chem 2015; 290: 20448-20454.

10 Wang T, Chen M, Liu L, Cheng H, Yan YE, Feng YH et al. Nicotine induced CpG methylation of Pax6 binding motif in StAR promoter reduces the gene expression and cortisol production. Toxicol Appl Pharmacol 2011; 257: 328-337.

11 Xia K, Wu L, Liu X, Xi X, Liang D, Zheng D et al. Mutation in PITX2 is associated with ring dermoid of the cornea. J Med Genet 2004; 41: e129.

12 Dar P, Javed AA, Ben-Yishay M, Ferreira JC, Paterson AD, Gross SJ et al. Potential mapping of corneal dermoids to Xq24-qter. J Med Genet 2001; 38: 719-723.

13 Tasse C, Bohringer S, Fischer S, Ludecke HJ, Albrecht B, Horn D et al. Oculo-auriculo-vertebral spectrum (OAVS): clinical evaluation and severity scoring of 53 patients and proposal for a new classification. Eur J Med Genet 2005; 48: 397-411.

14 Stark DJ, Gilmore DW, Vance JC, Pearn JH. A corneal abnormality associated with trisomy 8 mosaicism syndrome. Br J Ophthalmol 1987; 71: 29-31.

15 Scott JA, Howard PJ, Smith PA, Fryer A, Easty DL, Patterson A et al. Histopathology and molecular cytogenetics of a corneal 
opacity associated with the trisomy 8 mosaic syndrome (46,XY /47,XY, +8). Cornea 1997; 16: 35-41.

16 Wang R, Martinez-Frias ML, Graham JM Jr. Infants of diabetic mothers are at increased risk for the oculo-auriculovertebral sequence: a case-based and case-control approach. J Pediatr 2002; 141: 611-617.

17 Acs N, Banhidy F, Horvath-Puho E, Czeizel AE. Population-based case-control study of the common cold during pregnancy and congenital abnormalities. Eur J Epidemiol 2006; 21: 65-75.

18 Vogt G, Puho E, Czeizel AE. Population-based case-control study of isolated congenital cataract. Birth Defects Res A Clin Mol Teratol 2005; 73: 997-1005.

19 Blanco-Munoz J, Lacasana M, Borja-Aburto VH. Maternal miscarriage history and risk of anencephaly. Paediatr Perinat Epidemiol 2006; 20: 210-218.

20 Wells MD. Pregnancy-induced hypertension and congenital hearing loss. Int J Pediatr Otorhinolaryngol 1991; 22: 39-47.

21 Karkinen J, Saxen L. Maternal influenza, drug consumption, and congenital defects of the central nervous system. Am J Obstet Gynecol 1974; 118: 815-818.

22 Taranenko LA, Malutina NN. [Congenital abnormalities in children whose parents were exposed to methanol and formaldehyde]. Med Tr Prom Ekol 2012; 12: 33-35.

23 Oliveira NP, Moi GP, Atanaka-Santos M, Silva AM, Pignati WA. Congenital defects in the cities with high use of pesticides in the state of Mato Grosso, Brazil. Cien Saude Colet 2014; 19: 4123-4130.

24 Clayton D, McKeigue PM. Epidemiological methods for studying genes and environmental factors in complex diseases. Lancet 2001; 358: 1356-1360.

25 Sharma R. Revision of Prasad's social classification and provision of an online tool for real-time updating. South Asian J Cancer 2013; 2: 157.

26 Hosmer DW, Lemeshow S. Applied Logistic Regression. John Wiley \& Sons, Inc.: New York, 2000.

27 Luteijn JM, Brown MJ, Dolk H. Influenza and congenital anomalies: a systematic review and meta-analysis. Hum Reprod 2014; 29: 809-823.

28 Zhang J, Savitz DA, Schwingl PJ, Cai WW. A case-control study of paternal smoking and birth defects. Int J Epidemiol 1992; 21: 273-278.

29 Wasserman CR, Shaw GM, O'Malley CD, Tolarova MM, Lammer EJ. Parental cigarette smoking and risk for congenital anomalies of the heart, neural tube, or limb. Teratology 1996; 53: 261-267.

30 Deng K, Liu Z, Lin Y, Mu D, Chen X, Li J et al. Periconceptional paternal smoking and the risk of congenital heart defects: a case-control study. Birth Defects Res A Clin Mol Teratol 2013; 97: 210-216.

31 Thom DH, Nelson LM, Vaughan TL. Spontaneous abortion and subsequent adverse birth outcomes. Am J Obstet Gynecol 1992; 166: 111-116.

32 Goldenberg RL, Mayberry SK, Copper RL, Dubard MB, Hauth JC. Pregnancy outcome following a second-trimester loss. Obstet Gynecol 1993; 81: 444-446.

33 Maignien C, Nguyen A, Dussaux C, Cynober E, Gonzales M, Carbonne B. Outcome of pregnancy following second- or third-trimester intrauterine fetal death. Int J Gynaecol Obstet 2014; 127: 275-278.

34 Srinivas SK, Ernst LM, Edlow AG, Elovitz MA. Can placental pathology explain second-trimester pregnancy loss and subsequent pregnancy outcomes? Am J Obstet Gynecol 2008; 199: 402.e1-5.

35 Kovo M, Schreiber L, Bar J. Placental vascular pathology as a mechanism of disease in pregnancy complications. Thromb Res 2013; 131: S18-S21.

36 Tolosa JM, Parsons KS, Hansbro PM, Smith R, Wark PA. The placental protein syncytin-1 impairs antiviral responses and exaggerates inflammatory responses to influenza. PLoS One 2015; 10: e0118629.

37 Lim R, Sobey CG. Maternal nicotine exposure and fetal programming of vascular oxidative stress in adult offspring. Br J Pharmacol 2011; 164: 1397-1399.

38 Zdravkovic T, Genbacev O, McMaster MT, Fisher SJ. The adverse effects of maternal smoking on the human placenta: a review. Placenta 2005; 26(Suppl A): S81-S86.

39 Li G, Xu F, Zhu J, Krawczyk M, Zhang Y, Yuan J et al. Transcription factor Paired Box 6 controls limbal stem cell lineage in development and disease. J Biol Chem 2015; 290: 20448-20454.

40 Oberg M, Jaakkola MS, Woodward A, Peruga A, Pruss-Ustun A. Worldwide burden of disease from exposure to second-hand smoke: a retrospective analysis of data from 192 countries. Lancet 2011; 377: 139-146.

41 Oliver A, Overton C. Diagnosis and management of miscarriage. Practitioner 2014; 258: 25-28.

42 Mansour AM, Barber JC, Reinecke RD, Wang FM. Ocular choristomas. Surv Ophthalmol 1989; 33: 339-358.

Supplementary Information accompanies this paper on Eye website (http://www.nature.com/eye) 